\title{
Laser Cooling of Molecular Anions
}

\author{
Pauline Yzombard, ${ }^{1}$ Mehdi Hamamda, ${ }^{1}$ Sebastian Gerber, ${ }^{2}$ Michael Doser, ${ }^{2}$ and Daniel Comparat ${ }^{1}$ \\ ${ }^{1}$ Laboratoire Aimé Cotton, CNRS, Université Paris-Sud, ENS Cachan, Bâtiment 505, 91405 Orsay, France \\ ${ }^{2}$ CERN, European Laboratory for Particle Physics, 1211 Geneva, Switzerland
}

(Received 27 November 2014; published 27 May 2015)

\begin{abstract}
We propose a scheme for laser cooling of negatively charged molecules. We briefly summarize the requirements for such laser cooling and we identify a number of potential candidates. A detailed computation study with $\mathrm{C}_{2}{ }^{-}$, the most studied molecular anion, is carried out. Simulations of 3D laser cooling in a gas phase show that this molecule could be cooled down to below $1 \mathrm{mK}$ in only a few tens of milliseconds, using standard lasers. Sisyphus cooling, where no photodetachment process is present, as well as Doppler laser cooling of trapped $\mathrm{C}_{2}{ }^{-}$, are also simulated. This cooling scheme has an impact on the study of cold molecules, molecular anions, charged particle sources, and antimatter physics.
\end{abstract}

DOI: 10.1103/PhysRevLett.114.213001

PACS numbers: $37.10 . \mathrm{Mn}, 37.10 . \mathrm{Rs}$

Molecular anions play a central role in a wide range of fields: from the chemistry of highly correlated systems [1-4] to atmospheric science to the study of the interstellar medium [5-9]. However, it is currently very difficult to investigate negative ions in a controlled manner at the ultracold temperatures relevant for the processes in which they are involved. Indeed, at best, temperatures of a few kelvin have been achieved using supersonic beam expansion methods or trapped particles followed by electron cooling, buffer gas cooling, or resistive cooling [10-14]. The ability to cool molecular anions to subkelvin temperatures would finally allow investigation of their chemical and physical properties at energies appropriate to their interactions. Furthermore, anionic molecules at millidegree kelvin temperatures can also play an important role in (anti) atomic physics, where copious production of subkelvin antihydrogen atoms currently represents the dominant challenge in the field. Sympathetic cooling of antiprotons via laser-cooled atomic negative ions that are simultaneously confined in the same ion trap has been proposed as a method to obtain subkelvin antiprotons [15]. As an alternative to this yet-to-be-realized procedure, ultracold molecular anions could replace atomic anions in this scheme, and would thus facilitate the formation of ultracold antihydrogen atoms. More generally, cooling even a single anion species would be the missing tool to cool any other negatively charged particles (electrons, antiprotons, anions) via sympathetic cooling.

In this Letter, we present a realistic scheme for laser cooling of molecular anions to millidegree kelvin temperatures. Laser cooling of molecules has been achieved only for a very few neutral diatomic molecules $(\mathrm{SrF}, \mathrm{YO}, \mathrm{CaF})$

Published by the American Physical Society under the terms of the Creative Commons Attribution 3.0 License. Further distribution of this work must maintain attribution to the author(s) and the published article's title, journal citation, and DOI.
[16-18]. Furthermore, even if well established for neutral atoms and atomic cations, laser cooling techniques have so far never been applied to anions [19]. This is because in atomic anions, the excess electron is only weakly bound by quantum-mechanical correlation effects. As a result, only a few atomic anions are known to exhibit electricdipole transitions between bound states: $\mathrm{Os}^{-}, \mathrm{La}^{-}$, and $\mathrm{Ce}^{-}$[20-22].

For molecules the situation is quite different because their electric dipole can bind an extra electron, and even dianions have been found to exist [23]. For instance, polar molecules with a dipole exceeding 2.5D exhibit dipolebound states. Highly dipolar molecules such as $\mathrm{LiH}^{-}, \mathrm{NaF}^{-}$ or $\mathrm{MgO}^{-}$possess several such dipole-bound states [24,25], and valence anionic states exist as well. For simplicity, in this Letter we only focus on diatomic molecules, even if the Sisyphus laser cooling techniques proposed here can also be applied to trapped polyatomic molecules [26]. In the Supplemental Material, Table I [27], we present a review of most of the experimental as well as theoretical studies of diatomic anions, with useful references, if further studies are required.

The first excited state (and sometimes even the ground state) of many molecular anions lies above their neutralization threshold, such as in the case of $\mathrm{H}_{2}^{-}, \mathrm{CO}^{-}, \mathrm{NO}^{-}$, $\mathrm{N}_{2}{ }^{-}, \mathrm{CN}^{-}$or most of systems with 3,4 , or 11 outermost electrons. For this reason, these anions are not stable against autodetachment processes and exhibit pure rovibrational transitions with $\sim 100 \mathrm{~ms}$ lifetimes. Even if such long-lived states can still be of interest for Sisyphus cooling, for narrow-line cooling, or for Doppler laser cooling in traps $[26,28]$, pure electronic transitions are preferred for rapid laser cooling. Transitions of $\sim 100 \mathrm{~ns}$ lifetime can be found between well-separated electronic states (typically $B \leftrightarrow X$ states), whereas transitions in the infrared region between electronic states (typically $A \leftrightarrow X$ states) have longer lifetimes of $\sim 100 \mu$ s. Some challenges 
TABLE I. Diatomic anions with nine outermost electrons, listed in terms of groups of atoms. A more complete table with all diatomic molecules, and references, is given in the Supplemental Material [27]. They are isoelectronic to the neutral molecules $\mathrm{CN}$ and $\mathrm{SrF}$ with the same $X^{2} \Sigma^{+} A^{2} \Pi B^{2} \Sigma^{+}$electronic structure with sometimes stable quartet excited states.

\begin{tabular}{ll}
\hline \hline Group & Example \\
\hline I-VII & $\mathrm{LiF}^{-} \mathrm{LiCl}^{-} \mathrm{NaF}^{-} \mathrm{NaCl}^{-} \mathrm{MnH}^{-}\left(X^{6} \Delta\right)$ \\
II-VI & $\mathrm{ZnO}^{-} \mathrm{BeO}^{-} \mathrm{MgO}^{-} \mathrm{ZnF}^{-}$ \\
III-V & $\mathrm{BP}^{-} \mathrm{AlN}^{-} \mathrm{AlP}^{-} \mathrm{AlAs}^{-} \mathrm{GaP}^{-} \mathrm{InP}^{-} \mathrm{GaAs}^{-}$ \\
& $\mathrm{BN}^{-} \mathrm{GaN}^{-}$ \\
IV-IV & $\mathrm{C}_{2}^{-} \mathrm{Si}_{2}^{-} \mathrm{CSi}^{-}(B$ unstable $) \mathrm{Sn}_{2}^{-} \mathrm{Pb}_{2}^{-} \mathrm{SnPb}^{-}$ \\
& $\left(X^{2} \Pi\right)$ \\
\hline \hline
\end{tabular}

in laser-cooling molecular ions have been described in [29]. For direct laser cooling of neutral diatomic molecules, a key ingredient is a good branching ratio, i.e., FranckCondon factor (FC factor), between vibrational levels [SrF, YO, CaF all have more than a $98 \%$ branching ratio on the $A^{2} \Pi_{1 / 2}\left(v^{\prime}=0, J^{\prime}=1 / 2\right) \leftarrow X^{2} \Sigma\left(v^{\prime \prime}=0, N^{\prime \prime}=1\right)$ transition]. Even with these considerations, the choice of the most suitable candidate is not obvious and is a compromise between using fast electronic transitions and choosing extremely good FC factors. Indeed, molecules with good FC factors can be found for weak dipole-valence bound transitions or for anionic molecules with 6 or 12 outermost electrons but with forbidden dipole transitions. FC factors greater than $70 \%$ can be found in systems with 8,14 , or higher numbers of outermost electrons or in molecules which include $\mathrm{Li}$ or $\mathrm{Al}$ atoms [30,31]. Unfortunately the corresponding transitions are often in the deep infrared region. The best compromise seems to be the systems with nine outermost electrons having $X\left(\sigma^{1} \pi^{4}\right)^{2} \Sigma_{(g)}^{+}, A\left(\sigma^{2} \pi^{3}\right)^{2} \Pi_{(u)}, B\left(\sigma^{2} \pi^{4}\right)^{2} \Sigma_{(u)}^{+}$levels. A list of such systems is given in Table I.

Clearly the most studied system is $\mathrm{C}_{2}{ }^{-}$, with a perfectly known spectrum. $\mathrm{C}_{2}{ }^{-}$exhibits a $B^{2} \Sigma\left(v^{\prime}=0\right) \leftrightarrow$ $X^{2} \Sigma\left(v^{\prime \prime}=0\right)$ system and a $A^{2} \Pi_{1 / 2}\left(v^{\prime}=0\right) \leftrightarrow X^{2} \Sigma\left(v^{\prime \prime}=0\right)$ system with $72 \%$ and $96 \%$ branching ratio, respectively. Besides, this anion has the notable advantage of not presenting any hyperfine structure. As a potential further benefit of this system, we mention that through laser photodetachment of cold $\mathrm{C}_{2}{ }^{-}$, we could produce cold $\mathrm{C}_{2}$ molecules, important in combustion physics and astrophysics. Even if more studies on laser cooling are needed, $\mathrm{C}_{2}$ looks like a suitable candidate to be further laser cooled near $240 \mathrm{~nm}$ on the $0-0$ Mulliken band $\left(d^{1} \Sigma_{u}^{+} \leftarrow X^{1} \Sigma_{g}^{+}\right)$, which has an extremely favorable branching ratio of $99.7 \%$ [32].

We will therefore concentrate on $\mathrm{C}_{2}{ }^{-}$as a benchmark to study laser cooling of anionic molecules. Note, however, that several other molecules, such as $\mathrm{BN}^{-}$or $\mathrm{AlN}^{-}$, may be used as well. They offer very similar structure probabilities with
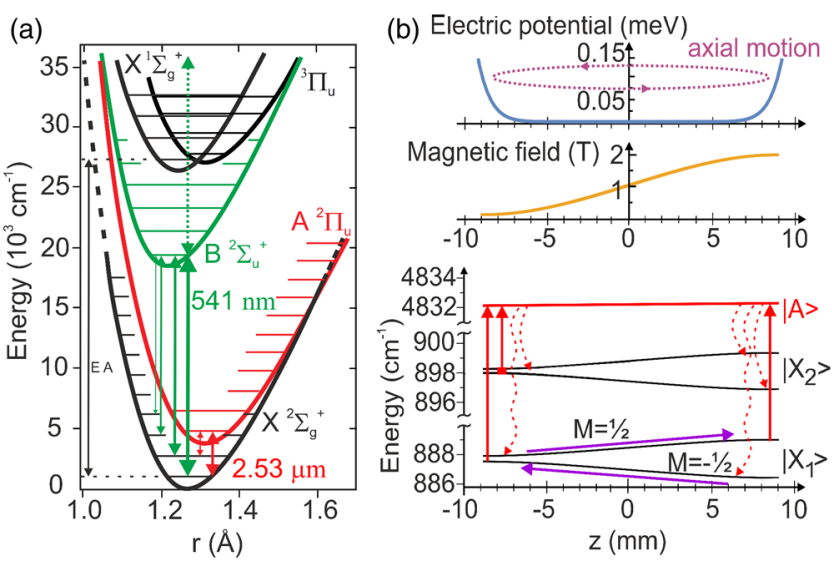

FIG. 1 (color online). $\mathrm{C}_{2}{ }^{-}$level structure and laser cooling transitions. (a) Electronic and vibrational levels including the photodetachment threshold of neutral $\mathrm{C}_{2}\left({ }^{1} \Sigma_{g}^{+},{ }^{3} \Pi_{u}\right)$, from [36]. The widths of the arrows are proportional to the transition strengths given by the FC. The green dotted line indicates that photodetachment can occur from the $B$ state due to a second photon absorption. (b) Detail of the Sisyphus cooling principle with a zoom on the $X \leftrightarrow A$ energies: $\left|X_{1}\right\rangle=X\left(v^{\prime \prime}=0, N^{\prime \prime}=0\right)$ and $\left|X_{2}\right\rangle=X\left(v^{\prime \prime}=0, N^{\prime \prime}=2\right),|A\rangle=A\left(v^{\prime}=0, N^{\prime}=1, J^{\prime}=1 / 2\right)$. A Penning-like trap is presented in the upper part. A magnetic gradient field creates the Sisyphus potential hill. The corresponding Zeeman effect on the $\mathrm{C}_{2}{ }^{-}$internal level states is plotted in the lower part. Laser excitations and spontaneous decays are, respectively, illustrated with red solid line and red dashed line.

better FC factors (higher than 98\%) but with a $B^{2} \Sigma \rightarrow A^{2} \Pi$ decay channel that is absent in the homonuclear case of $\mathrm{C}_{2}^{-}$. Contrary to $\mathrm{C}_{2}^{-}$, heteronuclear molecules present a closed rotational level scheme. However, further spectroscopic studies are clearly required for such systems, as well as for other promising ones, such as metal-oxide systems $\left(\mathrm{FeO}^{-}, \mathrm{NiO}^{-}\right)$or hydride ones $\left(\mathrm{CoH}^{-}\right)$.

In order to study laser cooling of $\mathrm{C}_{2}^{-}$we have performed three types of simulations. The first one is "standard" laser cooling in the gas phase (thus with no strong external fields present); the second one is laser cooling in a Paul trap; and the last one is Sisyphus cooling of trapped ions in a Penning trap. The simulations are performed with the $\mathrm{C}++$ code described in [28], which now also includes full $N$-body space charge effects [33]; the Lorentz force is solved by using Boris-Buneman integration algorithms [34,35]. Briefly, a kinetic Monte Carlo algorithm gives the exact time of events (absorption or emission of light) when solving the rate equations to study laser excitation of the molecules under the effect of Coulomb, light scattering, dipolar, Stark, and Zeeman forces. The $\mathrm{C}_{2}{ }^{-}$energy levels and required laser transitions are shown in Fig. 1(a).

The lifetime of the $B$ state is $75 \mathrm{~ns}[37,38]$ and wavelengths from its first vibrational level to the $X$ state vibrational levels are 541, 598, 667, 753, 863, 1007, $1206 \mathrm{~nm}$ with a corresponding transition strength probability of $72,23,5,0.8,0.1,2 \times 10^{-4}, 3 \times 10^{-5}, 4 \times 10^{-6}$ 
(percentage of the FC), from $[39,40]$. The lifetime of the $A$ state is $50 \mu$ s with wavelengths of $2.53 \mu \mathrm{m}$ (branching ratio of $96 \%$ ) and $4.57 \mu \mathrm{m}$ (branching ratio of $4 \%$ ). In order to close the rotational transition cycle, two lasers are required for each of these vibrational transitions to couple $X\left(N^{\prime \prime}=0,2\right)$ to $A\left(N^{\prime}=1, J^{\prime}=1 / 2\right)$, see Fig. 1(b).

For our simulations, the temperature along one axis is calculated from the deviation of $50 \%$ of the central velocities' histogram. The so-called 3D temperature $\left(T_{3 \mathrm{D}}\right)$ is the quadratic mean of the three one-dimensional temperatures $(x, y, z)$.

For our first simulation, we study a possible experiment based on deceleration of an anionic beam. In contrast to neutral cold molecules which are difficult to produce at low velocity, and in spite of the development of techniques such as velocity filtering, buffer gas cooling or decelerators (see list in [28]), an anionic beam can be brought to a standstill very easily by an electric field [41]. Indeed, a typical beam of $\mathrm{C}_{2}{ }^{-}$has a current of $1 \mathrm{nA}$ and is emitted at $1000 \mathrm{eV}$ with an energy dispersion of $1 \mathrm{eV}$ [42]. A 1000 volt potential box can thus decelerate such a beam. Furthermore, due to the energy dispersion of the beam, the stability of this voltage power supply is not critical. Typically $1 / 100000$ of the anions (i.e., $0.01 \mathrm{pA}$ current) will be decelerated inside the box to below an energy of $0.01 \mathrm{meV}$. This corresponds roughly to $0.1 \mathrm{~K}$, which is within the capture range of molasses cooling.

Thus, we propose a very simple deceleration scheme using a grounded vacuum chamber through which the $1000 \mathrm{eV}$ beam propagates; this is followed by a chamber at $900 \mathrm{~V}$ with a $3 \mathrm{~mm}$ radius hole which focuses the beam (1 cm downstream) to a smaller hole of $R \sim 0.3 \mathrm{~mm}$ radius of an innermost chamber at $1000 \mathrm{~V}$. The chambers could be made of transparent conducting film (such as indium tin oxide) or as grids to allow laser cooling. In this final chamber the electric field decreases as $\sim 0.2(z / R)^{-3}$ along the propagation axis $z$ [43] such that at $1 \mathrm{~cm}$ the Lorentz force becomes negligible compared to the laser cooling force that is typically $\hbar k \Gamma / 10 \sim 10^{-20} \mathrm{~N}$, corresponding to an acceleration of $10^{5} \mathrm{~m} \mathrm{~s}^{-2}$.

We simulate such a 3D molasses cooling with several $\mathrm{cw}$ lasers, all with $0.5 \mathrm{~W}$ power and $2 \mathrm{~cm}$ waists and wavelengths $10 \mathrm{MHz}$ red detuned from the transitions (Fig. 1). We consider here a cooling on the $X\left(v^{\prime \prime}=0, N^{\prime \prime}=2\right) \leftrightarrow$ $B\left(v^{\prime}=0, N^{\prime}=0\right)$ transition. For the $3 \mathrm{D}$ cooling we thus have six lasers on the $X\left(v^{\prime \prime}=0, N^{\prime \prime}=2\right) \leftrightarrow B\left(v^{\prime}=0\right.$, $\left.N^{\prime}=0\right)$ transition, two repumping counterpropagating lasers on the $X\left(v^{\prime \prime}=0, N^{\prime \prime}=0\right) \leftrightarrow B\left(v^{\prime}=0, N^{\prime}=0\right)$, plus extra repumping lasers: four lasers for each higher vibrational level of the ground state $X$ up to $v^{\prime \prime}=4$ (these lasers are counterpropagating along $\pm Z$ ). We start with 150 particles at $80 \mathrm{mK}$ distributed following a spherical Gaussian distribution $(\sigma=4 \mathrm{~mm})$. The result is indicated in Fig. 2(a). The first increase at $1 \mathrm{~ms}$ is due to the conversion of the Coulomb potential energy into kinetic

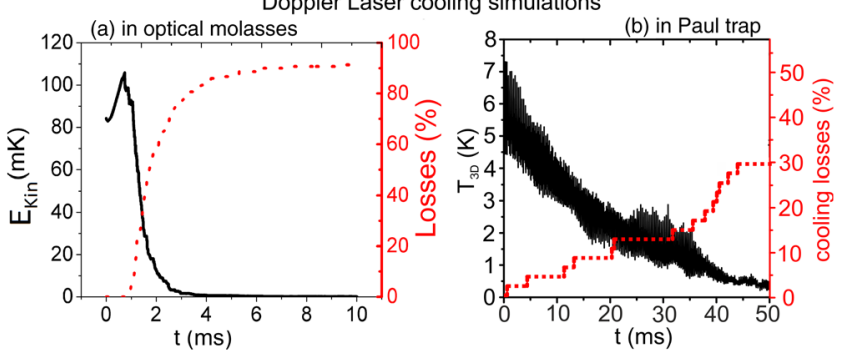

FIG. 2 (color online). Doppler laser cooling results, cycled on the $X \leftrightarrow B$ transition. (a) Deceleration and 3D molasses cooling of $\mathrm{C}_{2}{ }^{-}$. Result of the 3D laser cooling simulation of an initial bunch of 150 anions at $80 \mathrm{mK}$ : The red dashed curve gives the population losses, corresponding to molecules which escape from the laser interaction area. Black curve: kinetic energy evolution (in temperature unit) of the remaining particles. (b) In black, evolution of the temperature and number of $\mathrm{C}_{2}{ }^{-}$initially at $5 \mathrm{~K}$ in a Paul trap. The red dashed curve gives the population losses, through photoionization or decays into high vibrational ground states $(v>4)$.

energy. Then, a fast cooling down to $1 \mathrm{mK}$ is observed, although many molecules escape from the laser waist interaction area. We find that the final temperature and losses depend on the initial density (initial Gaussian radius) because of space charge effects that counter the cooling. Note that we do not attempt trapping but only molasses cool here. Indeed, realizing a trap (MOT) would require a magnetic-field gradient producing a Lorentz force stronger than the laser trapping one, especially because the Zeeman effect of $B\left(v^{\prime}=0, N^{\prime}=0\right)$ is weak (quadratic behavior) [44]. To be feasible, this cooling method requires precooling down to a few hundred millidegree kelvins in order to avoid having too fast molecules.

In the second simulation we study particles in a Paul trap. For simplicity, we only consider motion in a harmonic pseudopotential well $V(\mathbf{r})=1 / 2 m \omega_{r}\left(x^{2}+y^{2}\right)+1 / 2 m \omega_{z}^{2}$ [45]. We chose $5 \mathrm{~K}$ as a typical starting temperature of the beam. This would correspond to having carried out a first cooling step, using, e.g., helium as a buffer gas. We simulate Doppler cooling on the $X \leftrightarrow B$ transitions, with a $600 \mathrm{MHz}$ red detuning, and a spectral laser bandwidth of $35 \mathrm{MHz}$. As previously, we consider repumping lasers from the different vibrational levels of the ground state $X$ up to $X\left(v^{\prime \prime}=4\right)$, as shown in Fig. 1(a). But in this case repumping lasers are only along $+Z$, since particles are trapped. Results are given in Fig. 2(b) where cooling down to $60 \mathrm{mK}$ is achieved within $50 \mathrm{~ms}$. As the photodetachment cross section of $\mathrm{C}_{2}{ }^{-}$for the $B$ state is unknown, we use the photodetachment cross section of $\mathrm{C}_{2}, \sigma \sim 10^{-17} \mathrm{~cm}^{2}$ [13] as a typical value. The photodetachment rate for the $B$ state is $I \sigma / h \nu=4.3 \mathrm{~s}^{-1}$ for $I=0.16 \mathrm{~W} / \mathrm{cm}^{2}$. Within $50 \mathrm{~ms}$ of cooling we loose only $3 \%$ of the molecules by photodetachment. To these photodetachment losses, we have to add $25 \%$ of decays to higher vibrational levels of the $X$ state. 

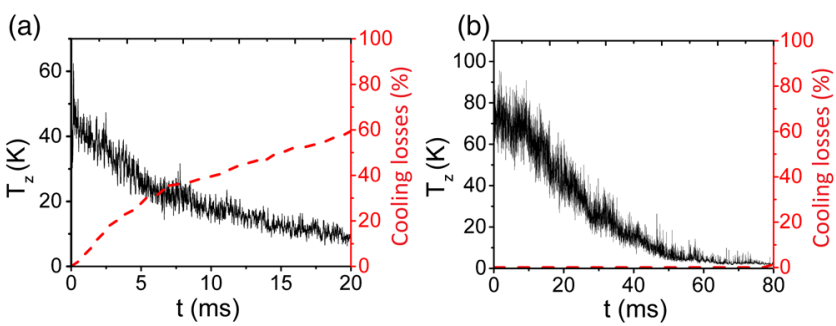

FIG. 3 (color online). Sisyphus cooling curves corresponding to the evolution of the axial temperature and number of $\mathrm{C}_{2}{ }^{-}$initially at 60 and $100 \mathrm{~K}$ in an inhomogenous Penning trap and cooled on the $X \leftrightarrow A$ transition, with (a) or without (b) repumping the $X\left(v^{\prime \prime}=1\right)$ losses.

The losses' evolution over time is shown in Fig. 2(b). Here, we would like to emphasize that for both molasses cooling and Paul trap simulations, we use a Doppler laser cooling process, with the same lasers. The Paul trap can thus serve as a first cooling and can be turned off for further cooling using the molasses cooling for low density clouds. Photodetachment and decays in higher vibrational $X$-state levels are thus similar. For the simulations of molasses cooling, however, losses due to the motion of molecules out of the laser's area are much more important than photodetachment or decay losses.

A solution to avoid the losses of $\mathrm{C}_{2}{ }^{-}$through photodetachment or decays into high vibrational levels of the ground state is to cool through the $A$ state. This cooling has very similar characteristics (linewidth, wavelength) to those of $\mathrm{La}^{-}$[22] but with a lighter particle and no photodetachment in the case of $\mathrm{C}_{2}{ }^{-}$.

Our third simulation thus concentrates on cooling and trapping in a $\sim 1.5 \mathrm{~cm}$ long Penning-like trap using Sisyphus-type cooling [28]. The principle is illustrated in Fig. 1(b): due to the axial motion induced by the electric trapping potential (300 $\mu$ s oscillation time in the simulation) in a Penning-like trap, particles move between the high $(2 \mathrm{~T})$ and low $(0.2 \mathrm{~T})$ magnetic fields at both ends of their axial range. A given particle is initially in the $X\left(v^{\prime \prime}=0, N^{\prime \prime}=0, M^{\prime \prime}=1 / 2\right)$ state; then, after an absorption followed by spontaneous emission, it decays towards the $X\left(v^{\prime \prime}=0, N^{\prime \prime}=0, M^{\prime \prime}=-1 / 2\right)$ state. More than $1 \mathrm{~K}$ is removed for each closed absorption-emission cycle, as the molecule continuously climbs the magnetic potential hill.

In the last 3D simulation we focus on the axial temperature, set initially at $100 \mathrm{~K}$ for a cloud of 200 anions. For our low density plasma there is no coupling between radial and axial motions, but the radial shape of the plasma reflects the inhomogeneous magnetic field. Two cooling lasers at 2.5351 and $2.5358 \mu \mathrm{m}$ with $100 \mathrm{MHz}$ spectral bandwidth each are detuned to be resonant at 0.2 and $2 \mathrm{~T}$, respectively, along the $X\left(v^{\prime \prime}=0, N^{\prime \prime}=0\right.$, $\left.M^{\prime \prime}=-1 / 2\right)_{0.2 T} \leftrightarrow A\left(v^{\prime}=0, N^{\prime}=1, J^{\prime}=1 / 2\right)_{0.2 T} \quad$ and $X\left(v^{\prime \prime}=0, N^{\prime \prime}=0, M^{\prime \prime}=1 / 2\right)_{2 T} \leftrightarrow A\left(v^{\prime}=0, N^{\prime}=1, J^{\prime}=\right.$ $1 / 2)_{2 T}$ transitions. The considered laser power is $0.05 \mathrm{~W}$ for a $1 \mathrm{~mm}$ waist. To avoid relying on too many lasers, we repump the losses in the $X\left(v^{\prime \prime}=0, N^{\prime \prime}=2\right)$ states with only one single broadband $(6000 \mathrm{MHz}, 0.05 \mathrm{~W})$ laser which addresses all the Zeeman-split sublevels, resonant at $0.2 \mathrm{~T}$.

Results are given in Fig. 3(a). In tens of ms, the axial temperature is cooled down from $60 \mathrm{~K}$ to few $\mathrm{K}$. The lost population mainly goes to $X\left(v^{\prime \prime}=1\right)$. As for all simulations, we load the trap using an initial (nonthermalized) Gaussian velocity distribution. The evolution of this nonequilibrium system leads to the high frequency velocity (and thus instantaneous temperature) fluctuations in both Fig. 2(b) and Fig. 3. We also present an alternative simulation, of which results are given in Fig. 3(b). Here, decays in the vibrational $X\left(v^{\prime \prime}=1\right)$ states are repumped: two lasers, at 4.574 and $4.593 \mu \mathrm{m}$, address the $\quad X\left(v^{\prime \prime}=1, N^{\prime \prime}=0\right) \leftrightarrow A\left(v^{\prime}=0, N^{\prime}=1, J^{\prime}=1 / 2\right)$ and $X\left(v^{\prime \prime}=1, N^{\prime \prime}=2\right) \leftrightarrow A\left(v^{\prime}=0, N^{\prime}=1, J^{\prime}=1 / 2\right)$. Both are resonant at $0.2 \mathrm{~T}$, with both spectral bandwidth of $6 \mathrm{GHz}$ and power of $0.1 \mathrm{~W}$.

This simulation requires two more lasers but has the advantage of cooling a greater part of the molecules [less than $0.5 \%$ of anions fall in the $X\left(v^{\prime \prime}=2\right)$ levels, within $80 \mathrm{~ms}$, in comparison to the $60 \%$ of losses within $20 \mathrm{~ms}$, for the first case without repumpers on $\left.X\left(v^{\prime \prime}=1\right)\right]$.

In conclusion, we have presented several possible deceleration and laser cooling schemes for anionic molecules, either in free space or trapped, by using Doppler or Sisyphus cooling, circumventing the problem of photodetachment. Working with traps can open many possibilities due to the long trapping times: it could enable restricting studies to only rovibrational transitions, or working with electronic transitions that have long spontaneous emission times. Furthermore, laser cooling of molecular anions followed by laser photodetachment could be used as a source for cold neutral molecules, or as an ideal source for electron bunches, since no Coulomb force due to ions will be present and ideal uniform elliptical density shapes could be realized $[46,47]$.

We are indebted to A. Kellerbauer, V. Kokoouline, O. Dulieu, C. Drag, M. Raoult, and H. Lignier for useful discussions. The research leading to these results has received funding from the European Research Council under Grant Agreement No. 277762 COLDNANO.

[1] M. T. Bell and T. P. Softley, Mol. Phys. 107, 99 (2009).

[2] O. Dulieu and C. Gabbanini, Rep. Prog. Phys. 72, 086401 (2009).

[3] L. D. Carr, D. DeMille, R. V Krems, and J. Ye, New J. Phys. 11, 055049 (2009).

[4] D. S. Jin and J. Ye, Chem. Rev. 112, 4801 (2012).

[5] X. Li and J. Paldus, Chem. Phys. Lett. 431, 179 (2006).

[6] J. Simons, J. Phys. Chem. A 112, 6401 (2008). 
[7] M. C. McCarthy, C. A. Gottlieb, H. Gupta, and P. Thaddeus, Astrophys. J. Lett. 652, L141 (2006).

[8] R. C. Fortenberry, IAU Symp. 297, 344 (2014).

[9] J. Simons, Annu. Rev. Phys. Chem. 62, 107 (2011).

[10] D. Gerlich, Phys. Scr. T59, 256 (1995).

[11] G. Gabrielse, A. Khabbaz, D. S. Hall, C. Heimann, H. Kalinowsky, and W. Jhe, Phys. Rev. Lett. 82, 3198 (1999).

[12] J. Deiglmayr, A. Goritz, T. Best, M. Weidemuller, and R. Wester, Phys. Rev. A 86, 043438 (2012).

[13] S. S. Kumar, D. Hauser, R. Jindra, T. Best, Š. Roučka, W. D. Geppert, T. J. Millar, and R. Wester, Astrophys. J. 776, 25 (2013).

[14] L. Biennier, S. Carles, D. Cordier, J.-C. Guillemin, S. D. Le Picard, and A. Faure, Icarus 227, 123 (2014).

[15] A. Kellerbauer and J. Walz, New J. Phys. 8, 45 (2006).

[16] E. S. Shuman, J. F. Barry, and D. DeMille, Nature (London) 467, 820 (2010).

[17] M. T. Hummon, M. Yeo, B. K. Stuhl, A. L. Collopy, Y. Xia, and J. Ye, Phys. Rev. Lett. 110, 143001 (2013).

[18] V. Zhelyazkova, A. Cournol, T. E. Wall, A. Matsushima, J. J. Hudson, E. A. Hinds, M. R. Tarbutt, and B. E. Sauer, Phys. Rev. A 89, 053416 (2014).

[19] A. Kellerbauer, A. Fischer, and U. Warring, Phys. Rev. A 89, 043430 (2014).

[20] L. Pan and D. R. Beck, Phys. Rev. A 82, 014501 (2010).

[21] C. W. Walter, N. D. Gibson, Y.-G. Li, D. J. Matyas, R. M. Alton, S. E. Lou, R. L. Field, D. Hanstorp, L. Pan, and D. R. Beck, Phys. Rev. A 84, 032514 (2011).

[22] C. W. Walter, N. D. Gibson, D. J. Matyas, C. Crocker, K. A. Dungan, B. R. Matola, and J. Rohlén, Phys. Rev. Lett. 113, 063001 (2014).

[23] S. N. Schauer, P. Williams, and R. N. Compton, Phys. Rev. Lett. 65, 625 (1990).

[24] K. R. Lykke, R. D. Mead, and W. C. Lineberger, Phys. Rev. Lett. 52, 2221 (1984).

[25] G. L. Gutsev, M. Nooijen, and R. J. Bartlett, Chem. Phys. Lett. 276, 13 (1997).

[26] M. Zeppenfeld, B. G. U. Englert, R. Glöckner, A. Prehn, M. Mielenz, C. Sommer, L. D. van Buuren, M. Motsch, and G. Rempe, Nature (London) 491, 570 (2012).

[27] See Supplemental Material at http://link.aps.org/ supplemental/10.1103/PhysRevLett.114.213001, which reviews most of the experimental as well as theoretical studies of the electronical states of diatomic anions depending on their number of valence electrons.

[28] D. Comparat, Phys. Rev. A 89, 043410 (2014).

[29] J. H. V. Nguyen, C. R. Viteri, E. G Hohenstein, C. D. Sherrill, K. R Brown, and B. Odom, New J. Phys. 13, 063023 (2011).

[30] A. I. Boldyrev, J. Simons, and P. von R. Schleyer, J. Chem. Phys. 99, 8793 (1993).

[31] G. L. Gutsev, P. Jena, and R. J. Bartlett, J. Chem. Phys. 110, 2928 (1999).

[32] O. Sorkhabi, D. D. Xu, V. M. Blunt, H. Lin, R. Price, J. D. Wrobel, and W. M. Jackson, J. Mol. Spectrosc. 188, 200 (1998).

[33] W. Dehnen and J. I. Read, Eur. Phys. J. Plus 126, 55 (2011).

[34] G. Zwicknagel, in Trapped Charged Particles and Fundamental Interactions, edited by F. Herfurth and H. K. Blaum, Lecture Notes in Physics Vol. 749 (Springer, Berlin, 2008), pp. 1-28.

[35] M. Toggweiler, J. Mol. Spectrosc. 273, 255 (2014).

[36] K. M. Ervin and W. C. Lineberger, J. Phys. Chem. 95, 1167 (1991).

[37] P. Rosmus, J. Phys. Chem. 80, 5085 (1984).

[38] T. Šdivcová and V. Špirko, Mol. Phys. 104, 1999 (2006).

[39] P. Jones, R. D. Mead, B. E. Kohler, S. D. Rosner, and W. C. Lineberger, J. Chem. Phys. 73, 4419 (1980).

[40] Y. Shan-Shan, Y. Xiao-Hua, L. Ben-Xia, K. Kakule, W. Sheng-Hai, G. Ying-Chun, L. Yu-Yan, and C. Yang-Qin, Chin. Phys. 12, 745 (2003).

[41] V. Rudnev and A. G. Ureña, Rev. Sci. Instrum. 84, 124102 (2013).

[42] U. Hefter, R. D. Mead, P. A. Schulz, and W. C. Lineberger, Phys. Rev. A 28, 1429 (1983).

[43] F. H. Read, N. J. Bowring, P. D. Bullivant, and R. R. A. Ward, Nucl. Instrum. Methods Phys. Res., Sect. A 427, 363 (1999).

[44] M. R. Tarbutt, New J. Phys. 17, 015007 (2015).

[45] C. B. Zhang, D. Offenberg, B. Roth, M. A. Wilson, and S. Schiller, Phys. Rev. A 76, 012719 (2007).

[46] O. J. Luiten, S. B. van der Geer, M. J. de Loos, F. B. Kiewiet, and M. J. van der Wiel, Phys. Rev. Lett. 93, 094802 (2004).

[47] D. H. Dubin and T. M. O’Neil, Rev. Mod. Phys. 71, 87 (1999). 